\title{
Integrated studies of the physical and mechanical properties of cotton fabric
}

\author{
Nodira Saydalieva ${ }^{*}$, Dilfuza Khudayberdieva $^{1}$, Feruza Isamukhamedova $^{2}$, and Illarion \\ Shin ${ }^{1}$ \\ ${ }^{1}$ Tashkent Institute of Textile and Light Industry, 100100 Tashkent, Uzbekistan \\ ${ }^{2}$ Uzbek State University of World Languages, 100138 Tashkent, Uzbekistan
}

\begin{abstract}
The nature of the adhesive forces of the K-4 preparation is manifested in the occurrence of hydrogen bonds between molecules, as a substance with a sufficiently developed ability to form hydrogen bonds with cellulose. By the method of a one-factor experiment, the regularities of the influence of the concentration of the preparation alkaline-hydrolyzed PAN product on shrinkage, the total opening angle and weight gain of the fabric were revealed. It was found that the concentration of the preparation, equal to $75 \mathrm{~g} / \mathrm{l}$, provides the minimum shrinkage and rinsability of the sizing, as well as at the same time the largest value of TOA.
\end{abstract}

\section{Introduction}

At the moment, the predominant method of finishing fabrics for household use is treatment with compositions containing high molecular weight compounds. An important role in improving the consumer properties of finished fabrics is played by the properties of polymer preparations used in compositions for finishing [1-3].

Acrylic compounds and derivatives of water-soluble acrylic compounds are widely used in chemical finishing as finishing preparations [4]. Due to the presence of a double bond and due to substituted groups, derivatives of acrylic compounds are able to interact with functional groups of macromolecules of the fibrous material due to adhesive forces, as well as polymerize, forming flexible polymer films $[5,6]$. Polyacrylonitrile latex in combination with cross-linking components is used as a finishing agent to give cotton fabrics low-shrink properties. The saponified polyacrylonitrile product is used as a sizing agent, thickener and a sizing component [7-10].

The purpose of this work is to establish the complex influence of the main factors on the physical and mechanical properties of cotton fabric in the process of final finishing [11].

Final finishing chemical operation based on the use of the K-4 preparation, which is an alkaline hydrolyzed PAN product. The nature of the adhesive forces of the K-4 preparation is manifested in the occurrence of hydrogen bonds between molecules, as a substance with a sufficiently developed ability to form hydrogen bonds with cellulose $[12,13]$.

\footnotetext{
* Corresponding author: nodira saydalieva@mail.ru
} 


\section{Materials and Methods}

The authors studied the influence of the concentration of the preparation of the alkalinehydrolyzed product PAN on the quality of the finish, expressed by shrinkage, by one-factor experiments. It was found that with an increase in the concentration of the finishing preparation of the alkaline-hydrolyzed product PAN, the tissue gain ranges from $5.6 \%$ to $11.4 \%$. It should be noted that with the preparation concentration increase up to $75 \mathrm{~g} / \mathrm{l}$ weight gain on fabric comprises $7,8 \%$ with minimum wash-off of the dressing, and at the same time the total opening angle of the finished fabric increases by $21 \%$ with a significant decrease in its shrinkage.

\section{Results and Discussion}

To establish the complex influence of various factors on the breaking load $(\mathrm{Pp}, \mathrm{N})$, elongation at break (lp, \%) and conditional stiffness (in conditional, $\mathrm{mcN} \cdot \mathrm{cm}^{2}$ ), the mathematical method of experiment planning was applied [10], which is used to study multifactor systems. As a mathematical model, which is a polynomial, we take the form of the response function $y=f\left(x_{1}, x_{2}, \ldots x_{n}\right)$. For three factors, the polynomial of the first degree is expressed by the following equation (1):

$$
\mathrm{y}=\mathrm{b}_{0}+\mathrm{b}_{1} \mathrm{x}_{1}+\mathrm{b}_{2} \mathrm{x}_{2}+\mathrm{b}_{3} \mathrm{x}_{3}+\mathrm{b}_{12} \mathrm{x}_{1} \mathrm{x}_{2}+\mathrm{b}_{13} \mathrm{x}_{1} \mathrm{x}_{3}+\mathrm{b}_{13} \mathrm{x}_{1} \mathrm{x}_{3}+\mathrm{b}_{23} \mathrm{x}_{2} \mathrm{x}_{3}+\mathrm{b}_{123} \mathrm{x}_{1} \mathrm{x}_{2} \mathrm{x}_{3}
$$

As input factors, $\mathrm{X}_{1}$ - K-4 (hydrolyzed PAN); $\mathrm{X}_{2}$ - PVA (polyvinyl acetate); and $\mathrm{X}_{3}$ heat setting $\left({ }^{\circ} \mathrm{C}\right)$ were taken. The levels and intervals of variation of the factors are presented in Table 1.

Table 1. Levels and intervals of factors variation.

\begin{tabular}{|c|c|c|c|c|c|c|}
\hline \multirow{2}{*}{$\#$} & \multirow{2}{*}{ Factors } & \multirow{2}{*}{ Code } & \multirow{2}{*}{$\begin{array}{l}\text { Variation } \\
\text { intervals }\end{array}$} & \multicolumn{3}{|c|}{ Levels of factors } \\
\cline { 5 - 7 } & & $\mathrm{Upper}+\mathbf{1}$ & Basic 0 & Lower -1 \\
\hline 1 & $\mathrm{~K}-4, \mathrm{~g} / 1$ & $\mathrm{X}_{1}$ & 25 & 100 & 75 & 50 \\
\hline 2 & $\mathrm{PVA}, \mathrm{g} / 1$ & $\mathrm{X}_{2}$ & 10 & 45 & 35 & 25 \\
\hline 3 & $\begin{array}{c}\text { Heat setting, } \\
{ }^{\circ} \mathrm{C}\end{array}$ & $\mathrm{X}_{3}$ & 20 & 160 & 140 & 120 \\
\hline
\end{tabular}

A full factorial experiment was used in the work, in which all possible combinations of $\mathrm{N}$ levels of factors (the number of experiments), determined by the following expression (2):

$$
N=m^{k}
$$

where, $\mathrm{m}$ - is the number of levels of each factor and $\mathrm{k}$ - is the number of factors.

For three factors, the full factorial experiment of type $2^{3}$ is represented by the matrix shown in Table 2. In order to eliminate errors, the experiments provided by the matrix are presented in a random sequence, i.e. according to the table of random numbers. The values of the coefficients of equation (1) for the breaking load $\mathrm{Pp}$ were found from the following dependencies:

1) on the warp of the fabric

absolute term $\mathrm{b}_{0}: b_{0}=\frac{1}{N} \sum_{J=1}^{N} y_{j}=\frac{1804}{8}=225,5$

regression coefficient characterizing linear effects 


$$
b_{i}=\frac{1}{N} \sum_{j=1}^{N} x_{i j} y_{j} ; \quad b_{1}=1,25 ; b_{2}=-22 ; b_{3}=7,75
$$

regression coefficients characterizing interaction effects

$b_{i j}=\frac{1}{N} \sum_{j=1}^{N} x_{i j} x_{l j} y_{j} ; b_{12}=-17,75 ; b_{13}=-27,88 ; b_{23}=11,25 ; b_{123}=-2 ;$

As a result of processing the experimental data, a regression equation with coded variables was obtained for the optimization parameter - breaking load Pp (3):

$$
\begin{gathered}
y=225,5+1,25 x_{1}-22 x_{2}+7,75 x_{3}-17,75 x_{1} x_{2}-27,88 x_{1} x_{3}+11,25 x_{2} x_{3}- \\
2 x_{1} x_{2} x_{3}
\end{gathered}
$$

2) on the weft of the fabric:

$$
y=188,25+1,25 x_{1}+13 x_{2}-2,75 x_{3}+11,5 x_{1} x_{2}+27,75 x_{1} x_{3}-41 x_{2} x_{3}+
$$

To check the statistical significance of the coefficients of the regression equations (3) and (4), the absolute values of the coefficients $/ \Delta b_{i} /, \mid \Delta b_{i j} /$ were compared with the confidence interval $\Delta b_{i}$. Having previously calculated the variance of the regression coefficients using this equation (5)

$$
S^{2}\left\{b_{i}\right\}=\frac{s_{y}^{2}}{N}
$$

We find the confidence interval of the coefficients from the dependence:

$$
\Delta b_{i}= \pm t S\left\{b_{i}\right\}
$$

where, $t$ is the table value of the Student's test, equal to 4.3 at a $5 \%$ significance level [6] and the number of degrees of freedom $f=2$.

The variance values $S_{y}^{2}$ of the optimization parameter (breaking load Pp) for the investigated fabric on the warp and weft, respectively, are equal to: 16.5 and 9.5.

Taking into account dependencies (5) and (6) we get:

1) on the warp:

$$
S^{2}\left\{b_{i}\right\}=\frac{S_{y}^{2}}{N}=\frac{16,5}{8}=2,06 \quad \Delta b_{i}= \pm t S\left\{b_{i}\right\}= \pm 4,3 \cdot \sqrt{2,06}= \pm 6,18
$$

2) on the weft:

$$
S^{2}\left\{b_{i}\right\}=1,1875 ; \Delta b_{i}= \pm 5,11
$$

The regression equations with statically significant coefficients are finally obtained (Table 2):

1) on the warp:

$$
y=225,5-22 x_{2}+7,75 x_{3}-17,75 x_{1} x_{2}-27,88 x_{1} x_{3}+11,25 x_{2} x_{3}
$$

2) on the weft:

$$
y=188,25+13 x_{2}-2,75 x_{3}+11,5 x_{1} x_{2}+27,75 x_{1} x_{3}-41 x_{2} x_{3}+11 x_{1} x_{2} x_{3}
$$




\section{ICECAE 2021}

Table 2. Matrix of experiments' planning and results.

\begin{tabular}{|c|c|c|c|c|c|c|c|c|c|c|c|c|c|c|}
\hline \multirow{3}{*}{$\#$} & \multirow{3}{*}{$\mathbf{x}_{0}$} & \multirow{3}{*}{$\mathbf{x}_{1}$} & \multirow{3}{*}{$\mathbf{x}_{2}$} & \multirow{3}{*}{$\mathbf{x}_{3}$} & \multirow{3}{*}{$\mathbf{x}_{1} \mathbf{x}_{2}$} & \multirow{3}{*}{$\mathbf{x}_{1} \mathbf{x}_{3}$} & \multirow{3}{*}{$\mathbf{x}_{2} \mathbf{x}_{3}$} & \multirow{3}{*}{$\mathbf{x}_{1} \mathbf{x}_{2} \mathbf{x}_{3}$} & \multicolumn{6}{|c|}{ Optimization parameters } \\
\hline & & & & & & & & & \multicolumn{2}{|c|}{ 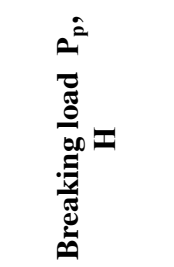 } & \multicolumn{2}{|c|}{ 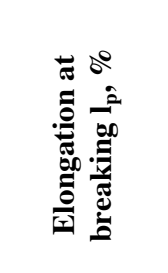 } & \multicolumn{2}{|c|}{ 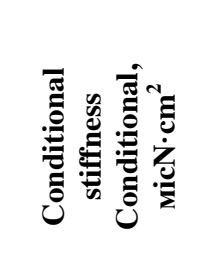 } \\
\hline & & & & & & & & & warp & weft & warp & weft & warp & weft \\
\hline 1 & + & - & - & + & + & - & - & + & 251 & 207 & 21 & 32 & 8445 & 6800 \\
\hline 2 & + & + & - & + & - & + & - & - & 237 & 220 & 20 & 25,5 & 2623 & 1849 \\
\hline 3 & + & - & + & + & - & - & + & - & 269 & 106 & 21 & 23,5 & 13105 & 10803 \\
\hline 4 & + & + & + & + & + & + & + & + & 176 & 209 & 20 & 26,5 & 8445 & 3210 \\
\hline 5 & + & - & - & - & + & + & + & - & 206 & 164 & 17,5 & 21,5 & 13552 & 5996 \\
\hline 6 & + & + & - & - & - & - & + & + & 296 & 110 & 21,5 & 22 & 9389 & 2842 \\
\hline 7 & + & - & + & - & - & + & - & + & 171 & 271 & 12,5 & 21 & 8223 & 2696 \\
\hline 8 & + & + & + & - & + & - & - & - & 198 & 219 & 32 & 22 & 9896 & 1270 \\
\hline
\end{tabular}

To test the hypothesis of the adequacy of the model represented by equations (5) and (6), we find the variance of the adequacy below (9):

$$
S_{a d}^{2}=\frac{\sum_{j=1}^{N}\left(y_{j-\widehat{y_{j}}}\right)^{2}}{f}
$$

where, $y_{j}$ - is the experimental value of the optimization parameter in the $\mathrm{j}$-m experiment; $\hat{y}_{j}$ - value of the optimization parameter in the $\mathrm{j}-\mathrm{m}$ experiment, calculated by the equation (7) and (8); $\mathrm{f}$ is the number of degrees of freedom, $\mathrm{f}=\mathrm{N}-(\mathrm{k}+1)$; and $\mathrm{k}$-number of factors equal to 3 .

The hypothesis of the adequacy of the model was tested using Fisher's F-test. For this, the calculated value of the criterion was found:

$$
F_{p}=\frac{S_{a d}^{2}}{S_{y}^{2}}=\frac{10}{16,5}=0,606
$$

At a 5\% significance level and the number of degrees of freedom for the numerator $f_{1}=$ 4 and for the denominator $f_{2}=2$, the tabular value of the criterion is $F_{T}=19.3$. Since $F_{p}<F_{T}$, the model represented by equation (7) is adequate. Let us check the adequacy of the model represented by equation (8):

$$
S_{a d}^{2}=\frac{\sum_{j=1}^{8}\left(y_{j}-\hat{y}_{i}\right)^{2}}{N-(k+1)}=\frac{23}{8-(3+1)}=5,75 ; \quad F_{p}=\frac{S_{\mathrm{az}}^{2}}{S_{y}^{2}}=\frac{5,75}{9,5}=0,605
$$


Therefore, the model is also adequate $\left(F_{p}<F_{T}\right)$. After processing the data of experimental studies for elongation at break (lp, \%) in the same sequence, the following regression equations with coded variables were obtained $(10,11)$ :

$$
\begin{gathered}
y=20,7+2,69 x_{1}+0.69 x_{2}-0.19 x_{3}+4.44 x_{1} x_{2}-3.19 x_{1} x_{3}-0.69 x_{2} x_{3}-1,94 x_{1} \\
x_{2} x_{3}(\text { warp) }(10) \\
y=24,3-0,25 x_{1}-x_{2}-2,63 x_{3}+1,19 x_{1} x_{2}-0,5 x_{1} x_{3}-0,88 x_{2} x_{3}+1,17 x_{1} x_{2} x_{3} \\
(\text { weft })(11)
\end{gathered}
$$

Taking into account the values of the confidence interval of the coefficients for the warp and weft, respectively, equal to $\Delta \mathrm{bi}= \pm 1.58, \Delta \mathrm{bi}= \pm 1.163$, we compose the regression equations with statically significant coefficients $(12,13)$ :

$$
\begin{array}{cc}
y=20,7+2,69 x_{1}+4,44 x_{1} x_{2}-3,19 x_{1} x_{3}-1,94 x_{1} x_{2} x_{3} & \text { (warp) (12) } \\
y=24,3+2,63 x_{3}+1,19 x_{1} x_{2}+1,13 x_{1} x_{2} x_{3} & \text { (weft) (13) }
\end{array}
$$

The verification of the adequacy of the mathematical model described by equations (12) and (13) is confirmed by comparing the calculated and tabular values of Fisher's Fcriterion, respectively, for the warp $\left(F_{p}<F_{T} ; 15.94<19.3\right)$ and the weft of the fabric $\left(F_{p}<F_{T} ; 14.12<19.3\right)$.

Processing the results of experimental studies for the optimization parameter conditional stiffness micN $\cdot \mathrm{cm}^{2}$ according to the above algorithm gave the following regression equations with coded variables $(14,15)$ :

$$
\begin{gathered}
y=9210-1622 x_{1}+707 x_{2}-1055 x_{3}+875 x_{1} x_{2}+999 x_{1} x_{3}+1913 x_{2} x_{3}- \\
584 x_{1} x_{2} x_{3}(\text { warp) (14) } \\
y=433-2141 x_{1}+827 x_{2}+1232 x_{3}-996 x_{1} x_{2}+1280 x_{1} x_{3}-546 x_{1} x_{2} x_{3} \\
(\text { weft) }(15)
\end{gathered}
$$

which adequately describe the model according to the F-criterion of Fisher. Thus, for equation (15), the following relation was obtained:

$$
\mathrm{F}_{\mathrm{p}}=15,2<\mathrm{F}_{\mathrm{T}}, \mathrm{F}_{\mathrm{T}}=19.3
$$

Thus, we obtained regression equations (7), (8), (12), (13), (14) and (15) with coded variables, which allow us to estimate the degree and nature of the influence of input factors and their paired interactions on optimization parameters.

Verification and confirmation of the hypothesis of the adequacy of mathematical models in the form of the obtained regression equations allow us to switch to the method of steep ascent according to Box-Wilson [8] to achieve the optimum region of the considered response functions. They choose for one factor, and for the rest $i$ calculate it using this equation (16):

$$
\Delta_{i}=\Delta_{l} \frac{b_{i} \varepsilon_{i}}{b_{l} \varepsilon_{l}}
$$


where, $\Delta_{l}$ is the selected step of movement for the factor $1 ; \Delta \mathrm{i}$ - movement step for the $\mathrm{i}$-th factor; $b_{i} ; b_{l}$ - regression coefficients of the $\mathrm{i}$-th and 1-th factors; and $\varepsilon_{i} ; \varepsilon_{l}$ - intervals of variation of the $i-$ th and $1-$ th factors.

The movement along the gradient starts from the zero point (the main level of the factor). Having calculated the step of movement for each factor, the conditions of "mental" experiments are found. Some thought experiments are carried out to test the results of a steep ascent. A steep ascent is terminated if the optimization conditions are found, and also if the constraint on the factor makes further movement along the gradient unreasonable.

Let us make a steep ascent (Table 3) along the response surface for the optimization parameter - breaking load (on the warp). A steep ascent begins on condition $\mathrm{x}_{1}=\mathrm{x}_{2}=\mathrm{x}_{3}=0$, which corresponds to the value of the input factors: $75 ; 35$ and 140 . Let us take the step of movement for the input factor $\mathrm{x}_{3}$ equal to $\Delta_{3}=5^{0} \mathrm{C}$. According to Equation 16, the step of movement was calculated for the factors $\mathrm{x}_{1}$ and $\mathrm{x}_{2}$ (Table 3 ):

$$
\Delta_{1}=\Delta_{3} \frac{b_{1} \varepsilon_{1}}{b_{3} \varepsilon_{3}}=5 \frac{(-1622) * 25}{1232 * 20}=-8,2285 ; \quad \Delta_{2}=\Delta_{3} \frac{b_{2} \varepsilon_{2}}{b_{3} \varepsilon_{3}}=5 \frac{707 * 10}{1232 * 20}=1,4347
$$

Table 3. Calculation of steep ascent for the optimization parameter - breaking load $\mathrm{Pp}$ (on the warp).

\begin{tabular}{|c|c|c|c|c|}
\hline Name & $\mathbf{x}_{\mathbf{1}}$ & $\mathbf{x}_{\mathbf{2}}$ & $\mathbf{x}_{\mathbf{3}}$ & $\mathbf{y}$ \\
\hline Basic level & 75 & 35 & 140 & - \\
\hline Coefficient $\mathrm{b}_{\mathrm{i}}$ & $-1,622$ & 707 & $-1,055$ & - \\
\hline Variation interval $\varepsilon_{1}$ & 25 & 10 & 20 & - \\
\hline $\mathrm{b}_{\mathrm{i}} \mathrm{x} \varepsilon_{1}$ & $-40,550$ & 7,070 & 21,100 & - \\
\hline Step $\Delta_{i}$ & -8.2285 & 1.4347 & 5 & - \\
\hline Rounded step & -8.23 & 1.43 & 5 & - \\
\hline Experiment 9 realized & 66.8 & 36.4 & 145 & 307 \\
\hline Experiment 10 realized & 58.5 & 37.8 & 150 & 311 \\
\hline Experiment 11 realized & 50.3 & 39.2 & 155 & 302 \\
\hline Experiment 12 thought & 42.1 & 40.6 & 160 & - \\
\hline
\end{tabular}

The maximum breaking load value (on the warp) was obtained in experiment No. 10, which was $\mathrm{Pp}=311 \mathrm{~N}$. Thus, the optimum region for breaking load was reached with the following values of the input factors: Preparation K-4 - $58.5 \mathrm{~g} / \mathrm{l}$ and PVA- $37.8 \mathrm{~g} / \mathrm{l}$; Heat setting $-150{ }^{\circ} \mathrm{C}$.

Thus, 11 tests were required in order to determine the optimal conditions for the final finishing of the cotton fabric, ensuring the maximum breaking load.

\section{Conclusions}

By the method of a one-factor experiment, the regularities of the influence of the concentration of the preparation of the alkaline-hydrolyzed product PAN on the shrinkage, the total opening angle and the weight gain of the finished tissue were revealed. It was found that the concentration of the drug, equal to $75 \mathrm{~g} / 1$, provides the minimum shrinkage and wash-off of the sizing, as well as at the same time the highest value of the total opening angle (TOA).

Using the method of multifactorial planning of the experiment, regression equations were obtained for various optimization parameters - breaking load, breaking elongation of the stiffness conditional depending on the input parameters. The steep ascent along the 
response surface provided the best experimental conditions for optimizing the breaking force of the tissue.

\section{References}

1. S. Mommer, J. Kurniadi, H. Keul, M. Moller, Applied Polymer Science 4, 74-79 (2020)

2. Y. Yang, Z. Guo, W. Huang, S. Zhang, J. Huang, H. Yang, Y. Zhou, Applied Surface Science 503, 11 (2020)

3. E. G. Polushin, O. V. Kozlova, A. S. Zakharchenko, V. E. Rumyantseva, Technology of the textile industry 3, 10 (2019)

4. A. E. Tretyakov, V. V. Safonov,.Chemical safety 2(1), 1014-1021 (2018)

5. P. Hwan, Ch. Hyung-Min, O. K. Wha, Cellulose 4, 3107-3119 (2014)

6. B. S. Batyrkhanova, L. V. Loginova, A. Zh. Kutzhanova, Bulletin of the Almaty Technological University 14(3), 71 - 77 (2015)

7. Ch. Debapriya, M. S. Inamdar, Indian J. Fibre and Text. Res. 38(1), 14-21 (2013)

8. M. G. Martosenko, L. V. Polishchuk, B. D. Semak, J. Commodity Vesnik 6, 72 - 79 (2013)

9. G. A. Ikhtiyarova, J. Tex. Prob. 1(12), 17 (2008)

10. M. Kh. Mirzakhmedova, D. B. Khudayberdieva, M. Z.Abdukarimova, G. K. Sodikova, J. Izvestiya VUZov series: Tech. of the tex. ind. 1, 014178 (2017)

11. D. B. Khudaiberdieva, Composition materials 4, 12 (2007)

12. N. Z. Saydalieva, D. B. Khudayberdieva, J. B. Suyunov, X. B. Sultonoyozov, J. of Advan. Res. in Sci. 6(10), 1214-1224 (2019)

13. A. A. Spriddinov, Planning an expert in the study of technological processes, 354 (Nauka Press, Moscow, 1981) 